\title{
Sensors for First Responder Applications
}

\author{
Angela M. Ervin, Ph.D. \\ ${ }^{1}$ U.S. Department of Homeland Security, Science and Technology Directorate \\ First Responders Group \\ Washington, DC 20528 USA \\ Angela.Ervin@hq.dhs.gov
}

\begin{abstract}
:
Department of Homeland Security Science and Technology Directorate funded research and development activities to meet first responder requirements in the area of sensors for detection of chemical vapors and opioids will be described.
\end{abstract}

Key words: Sensors, wearable badge, toxic industrial chemicals detection, fentanyl detection, vapor detection.

\section{Introduction}

The U.S. Department of Homeland Security (DHS) Science and Technology Directorate (S\&T) invests in research and development (R\&D) to develop technology solutions, knowledge products, methods and procedures to mitigate the adverse consequences resulting from a multitude of threats to the homeland, to include the release of chemical, biological and agricultural agents.

This presentation is an overview of funded R\&D activities within the S\&T First Responders Group (FRG) in the areas of chemical vapor detection and opioid detection. Working in close partnership with first responders at all levels, FRG identifies needs and requirements of the first responder community and then evaluates the use of existing or emerging technologies, knowledge products and standards to provide solutions for those identified needs. The focus of the FRG R\&D activities include: making first responders safer; helping first responders share data and criticial information; enhancing first responders voice communicate through interoperability; and engaging with first responders to ensure S\&T activities remain relevant.

Details of the three specific topic requirements to be discussed have been published.

Following are excerpts from those topics.

The first topic requests solutions for a device that is portable or handheld, that will detect and quantify levels of toxic gases, vapors, and particulates commonly found in the post-fire environment. The portable or hand-held device shall be intrinsically safe, small and lightweight with a goal of weighing less than 1 pound with a threshold of less than 3 pounds. It shall be able to work in normal heat and cold conditions; operating from between $140^{\circ} \mathrm{F}$ to $-30^{\circ} \mathrm{F}$ temperatures, water-resistant, and rugged enough to withstand a drop test of a minimum of 10 feet. The device shall be small (goal of 4 " $\times 3$ " $\times 1$ " with a threshold of 6" $\times 4$ " $\times 2$ ") and ideally be able to be adhered to or integrated with other First Responder Personal Protective Equipment (PPE), such as the Self-contained Breathing Apparatus (SCBA) or the Personal Alert Safety System (PASS). The device shall have a minimum of a 4-hour battery life and the batteries required to operate the system shall be commercially available. If rechargeable batteries are used, there should be an ability to swap out off-the-shelf batteries if there is no time to perform a recharge. In addition, the long-term sustainment for the device's sensors should allow for maintenance to be conducted by the user rather than require manufacturer involvement. The price goal should be less than USD $\$ 1,500$ per unit, with a threshold of less than USD $\$ 5,000$ per unit. The response time goal of the system should be less than 1 minute, with a threshold of less than 5 minutes. The device should provide the capability for the user to immediately know about present dangers, and include clear (preset) audible and visual alarm indicators. The display shall be readable in all light conditions and should 
display only relevant data such as an indication that the device is operating normally and/or information concerning a specific detection notification. The device should include displays for both current and cumulative exposure levels. The level of detection goal for the device shall be within Permissible Exposure Limits (PEL). The threshold for detection shall be Acute Exposure Guideline Level (AEGL) 2 at a 30-minute exposure.

The device shall, at a minimum, monitor and detect the following gases and particulates: Chlorine; Methane; Carbon Monoxide; Carbon Dioxide; Hydrogen; Hydrogen Sulfide; Hydrogen Chloride; Hydrogen Cyanide; Ammonia; Oxygen; Lower Explosive Limit; Sulfur Dioxide; Mercury; and detection scheme must include a particle counter with user settable alarm thresholds.

The second topic request solutions for a wearable sensor that is able to respond to multiple toxic industrial chemicals (TICs) with particular attention paid to cross sensitivity between target chemicals and effects of common interferents and environmental conditions to minimize false alarms. The wearable chemical sensor dimensions should not exceed $2 \times 2$ inches with a maximum of 0.5 inches in thickness ("badge"). Ultimately, the approach should be amenable to being configured as a button as part of a first responder uniform. The badge should have more than one type of alarm indicator (such as visible and audible alerts, visible and vibration alerts, etc). The response time of the sensor should have an objective of one second or a threshold of 30 seconds. The sensor badge should have a shelf like of at least twelve month (storage in package) and weigh less than $100 \mathrm{~g}$ including the batteries. The cost of the badge should be affordable (objective: USD \$30; threshold: USD \$50). If appropriate, the vendor should propose disposable sensing elements at less than USD \$5 per element with a reusable badge casing. The developed prototype badge should respond to at least four high priority TICs down to the permissible exposure limits (PEL) with negligible false alarm rate. Suggested TICs are provided below, in priority order: Chlorine; Carbon Monoxide; Hydrogen Sulfide; Carbon Dioxide; Nitrogen Dioxide; Methane; Ammonia; Hydrogen Cyanide; Phosphine; and Methyl Bromide.

The third topic requests solutions for a wearable sensor that is able to recognize fentanyl and its analogs in air and provide a signal warning to wearer. A wearable fentanyl sensor will be useful for monitoring DHS and First Responder personnel for the presence of harmful levels of these opioid compounds in the air so that protective measures can be taken before physical effects are seen such as drowsiness, dizziness, nausea and confusion. The device should signal the presence of fentanyl and its analogs within minutes of contact. (Threshold - 10 Minutes; Goal - 3 minutes). It should be small with the targeted goal of having a 2 inch square or smaller footprint. Detection of the fentanyl compound will be signaled both audibly and visually for the wearer. The fentanyl sensor should be selfpowered with a run time goal of 48 hours and minimal threshold of 24 hours. The power should be able to be replenished or replaced easily by the user with a cheap commerciallyavailable battery. Sensitivity and specificity are the most important specifications in relation to any detection device. The objective for sensitivity of the device in air is $1.6 \mathrm{mg}(\mathrm{min}) / \mathrm{m}^{3}$ ECt50 (effective concentration 50 percent) with a threshold of $3.3 \mathrm{mg}(\mathrm{min}) / \mathrm{m}^{3}$ ECt50. This level of detection would protect humans from a mild effective dose of fentanyl by the inhalation route. The wearable sensor will detect the current and emerging fentanyl synthetic opioids as demonstrated by recognition of fentanyl itself and fentanyl class compounds. Testing will demonstrate that potential "cutting agents" such as mannitol, lactose, dipyrone, baking soda, heroin, and other emerging cutting agents do not interfere with sensing of fentanyl class compounds even when they make up only 1 percent of the tested mixture by weight. The objective cost of the wearable fentanyl sensor is USD \$10 with a threshold cost of USD \$20.

During this presentation, the speaker will provide an overview of the DHS S\&T organization, describe ongoing research within FRG to provide solutions to referenced topic requirements, as well as opportunities and mechanisms of how to do business with DHS S\&T.

\section{References}

(1) https://www.osha.gov/dsg/annotated-pels; https://www.epa.gov/aegl

(2) https://sbir2.st.dhs.gov/portal/SBIR

(3) https://www.dhs.gov

(4) https://www.dhs.gov/science-and-technology/firstresponders-group 